\title{
Corrigendum: A High-Protein, Low Glycemic Index Diet Suppresses Hunger but Not Weight Regain After Weight Loss: Results From a Large, 3-Years Randomized Trial (PREVIEW)
}

Ruixin Zhu ${ }^{1}$, Mikael Fogelholm ${ }^{2}$, Thomas M. Larsen ${ }^{1}$, Sally D. Poppitt ${ }^{3}$, Marta P. Silvestre ${ }^{3,4}$, Pia S. Vestentoft ${ }^{1}$, Elli Jalo ${ }^{2}$, Santiago Navas-Carretero ${ }^{5,6,7}$, Maija Huttunen-Lenz ${ }^{8}$, Moira A. Taylor ${ }^{9,10}$, Gareth Stratton ${ }^{11}$, Nils Swindell ${ }^{11}$, Niina E. Kaartinen ${ }^{12}$, Tony Lam ${ }^{13}$, Teodora Handjieva-Darlenska ${ }^{14}$, Svetoslav Handjiev ${ }^{14}$, Wolfgang Schlicht ${ }^{15}$, J. Alfredo Martinez ${ }^{6,7,16,17}$, Radhika V. Seimon ${ }^{18}$, Amanda Sainsbury ${ }^{19}$, Ian A. Macdonald ${ }^{20}$, Margriet S. Westerterp-Plantenga ${ }^{21}$, Jennie Brand-Miller ${ }^{22}$ and Anne Raben ${ }^{1,23 *}$

\section{OPEN ACCESS}

Approved by:

Frontiers Editorial Office,

Frontiers Media SA, Switzerland

*Correspondence: Anne Raben ara@nexs.ku.dk

Specialty section: This article was submitted to Nutrition and Metabolism,

a section of the journal

Frontiers in Nutrition

Received: 05 July 2021 Accepted: 06 July 2021 Published: 23 July 2021

Citation:

Zhu R, Fogelholm M, Larsen TM, Poppitt SD, Silvestre MP, Vestentoft PS, Jalo $E$ Navas-Carretero S, Huttunen-Lenz $M_{\text {, }}$

Taylor MA, Stratton G, Swindell N, Kaartinen NE, Lam T, Handjieva-Darlenska T, Handjiev S, Schlicht W, Martinez JA, Seimon RV, Sainsbury A, Macdonald IA, Westerterp-Plantenga MS, Brand-Miller $J$ and Raben A (2021) Corrigendum: A High-Protein, Low

Glycemic Index Diet Suppresses Hunger but Not Weight Regain After Weight Loss: Results From a Large, 3-Years Randomized Trial (PREVIEW).

Front. Nutr. 8:736531.

doi: 10.3389/fnut.2021.736531
${ }^{1}$ Department of Nutrition, Exercise and Sports, Faculty of Science, University of Copenhagen, Copenhagen, Denmark, ${ }^{2}$ Department of Food and Nutrition, University of Helsinki, Helsinki, Finland, ${ }^{3}$ Human Nutrition Unit, School of Biological Sciences, Department of Medicine, University of Auckland, Auckland, New Zealand, ${ }^{4}$ Center for Health Technology Services Research, NOVA Medical School, Universidade Nova de Lisboa, Lisbon, Portugal, ${ }^{5}$ Centre for Nutrition Research, University of Navarra, Pamplona, Spain, ${ }^{6}$ Centro de Investigacion Biomedica en Red Area de Fisiologia de la Obesidad y la Nutricion (CIBEROBN), Madrid, Spain, ${ }^{7}$ disNA Instituto for Health Research, Pamplona, Spain, ${ }^{8}$ Institute for Nursing Science, University of Education Schwäbisch Gmünd, Schwäbisch Gmünd, Germany, ${ }^{9}$ Division of Physiology, Pharmacology and Neuroscience, School of Life Sciences, Queen's Medical Centre, Nottingham, United Kingdom, ${ }^{10}$ National Institute for Health Research (NIHR) Nottingham Biomedical Research Centre, Nottingham, United Kingdom, ${ }^{11}$ Applied Sports, Technology, Exercise and Medicine (A-STEM) Research Centre, Swansea University, Swansea, United Kingdom, ${ }^{12}$ Department of Public Health and Welfare, Finnish Institute for Health and Welfare, Helsinki, Finland, ${ }^{13}$ NetUnion sarl, Lausanne, Switzerland, ${ }^{14}$ Department of Pharmacology and Toxicology, Medical University of Sofia, Sofia, Bulgaria, ${ }^{15}$ Exercise and Health Sciences, University of Stuttgart, Stuttgart, Germany, ${ }^{16}$ Department of Nutrition and Physiology, University of Navarra, Pamplona, Spain, ${ }^{17}$ Precision Nutrition and Cardiometabolic Health Program, IMDEA (Madrid Institute for Advanced Studies)-Food Institute, CEI UAM + CSIC (Campus de Excelencia Internacional, Universidad Autónoma de Madrid + Consejo Superior de Investigaciones Científicas), Madrid, Spain, ${ }^{18}$ The Boden Collaboration for Obesity, Nutrition, Exercise, and Eating Disorders, Faculty of Medicine and Health, Charles Perkins Centre, The University of Sydney, Camperdown, NSW, Australia, ${ }^{19}$ School of Human Sciences (Exercise and Sports Science), Faculty of Science, The University of Western Australia, Crawley, WA, Australia, ${ }^{20}$ Division of Physiology, Pharmacology and Neuroscience, School of Life Sciences, Queen's Medical Centre, MRC/ARUK Centre for Musculoskeletal Ageing Research, ARUK Centre for Sport, Exercise and Osteoarthritis, National Institute for Health Research (NIHR) Nottingham Biomedical Research Centre, Nottingham, United Kingdom, ${ }^{21}$ Department of Nutrition and Movement Sciences, NUTRIM, School of Nutrition and Translational Research in Metabolism, Maastricht University, Maastricht, Netherlands, ${ }^{22}$ School of Life and Environmental Sciences and Charles Perkins Centre, The University of Sydney, Sydney, NSW, Australia, ${ }^{23}$ Steno Diabetes Center Copenhagen, Gentofte, Denmark

Keywords: obesity, pre-diabetes, satiety, desire to eat, low-energy diet, weight-loss maintenance

\section{A Corrigendum on}

A High-Protein, Low Glycemic Index Diet Suppresses Hunger but Not Weight Regain After Weight Loss: Results From a Large, 3-Years Randomized Trial (PREVIEW)

by Zhu, R., Fogelholm, M., Larsen, T. M., Poppitt, S. D., Silvestre, M. P., Vestentoft, P. S., et al. (2021). Front. Nutr. 8:685648. doi: 10.3389/fnut.2021.685648

In the published article, there was an error in affiliations 5, 6 and 7. Instead of " 5 Department of Nutrition, University of Navarra, Pamplona, Spain, 6 CIBERobn, Instituto de Salud 
Carlos III, Madrid, Spain, 7 Precision Nutrition Program, IMDEA Food, Campus de Excelencia Internacional, Universidad Autónoma de Madrid + Consejo Superior de Investigaciones Científicas, Madrid, Spain", it should be " 5 Centre for Nutrition Research, University of Navarra, Pamplona, Spain, 6 Centro de Investigacion Biomedica en Red Area de Fisiologia de la Obesidad y la Nutricion (CIBEROBN), Madrid, Spain, 7 IdisNA Instituto for Health Research, Pamplona, Spain”.

In the published article, there was an error regarding the affiliations for J. Alfredo Martinez. As well as having affiliations 6 and 7, they should also have "16 Department of Nutrition and Physiology, University of Navarra, Pamplona, Spain" and "17 Precision Nutrition and Cardiometabolic Health Program, IMDEA (Madrid Institute for Advanced Studies)-Food Institute, CEI UAM + CSIC (Campus de Excelencia Internacional, Universidad Autónoma de Madrid + Consejo Superior de Investigaciones Científicas), Madrid, Spain"

In the original article, there was an error. In the Conflict of Interest statement, "TLar is advisor for "Sense" diet program AS owns 50\% of the shares in Zuman International" should be "TLar is advisor for "Sense" diet program. AS owns $50 \%$ of the shares in Zuman International"
In the original article, there was an error. In the Conflict of Interest statement, "JB-M was President and Director of the Glycemic Index Foundation" should be "JB-M is President and Director of the Glycemic Index Foundation".

The authors apologize for these errors and state that these do not change the scientific conclusions of the article in any way. The original article has been updated.

Publisher's Note: All claims expressed in this article are solely those of the authors and do not necessarily represent those of their affiliated organizations, or those of the publisher, the editors and the reviewers. Any product that may be evaluated in this article, or claim that may be made by its manufacturer, is not guaranteed or endorsed by the publisher.

Copyright (c) 2021 Zhu, Fogelholm, Larsen, Poppitt, Silvestre, Vestentoft, Jalo, Navas-Carretero, Huttunen-Lenz, Taylor, Stratton, Swindell, Kaartinen, Lam, Handjieva-Darlenska, Handjiev, Schlicht, Martinez, Seimon, Sainsbury, Macdonald, Westerterp-Plantenga, Brand-Miller and Raben. This is an open-access article distributed under the terms of the Creative Commons Attribution License (CC BY). The use, distribution or reproduction in other forums is permitted, provided the original author(s) and the copyright owner(s) are credited and that the original publication in this journal is cited, in accordance with accepted academic practice. No use, distribution or reproduction is permitted which does not comply with these terms. 